\title{
The protein binding substance Ibuprofen does not affect the T1 time or partition coefficient in contrast-enhanced cardiovascular magnetic resonance
}

\author{
Nadine Kawel ${ }^{1 *}$, Francesco Santini ${ }^{2}$, Tanja Haas ${ }^{1}$, Johannes M Froehlich ${ }^{3}$ and Jens Bremerich ${ }^{1}$
}

\begin{abstract}
Background: Contrast enhanced cardiovascular magnetic resonance (CMR) with T1 mapping enables quantification of diffuse myocardial fibrosis. Various factors, however, can interfere with $\mathrm{T1}$ measurements. The purpose of the current study was to assess the effect of co-medication with a typical protein binding drug (Ibuprofen) on T1 values in vitro and in vivo.

Methods: 50 vials were prepared with different concentrations of gadobenate dimeglumine, Ibuprofen and human serum albumin in physiologic $\mathrm{NaCl}$ solution and imaged at 1.5T with a spin echo sequence at multiple TRs to measure $\mathrm{T} 1$ values and calculate relaxivities. 10 volunteers ( 5 men; $31 \pm 6.3$ years) were imaged at $1.5 \mathrm{~T}$. T1 values for myocardium and blood pool were determined for various time points after administration of $0.15 \mathrm{mmol} / \mathrm{kg}$ gadobenate dimeglumine using a modified look-locker inversion-recovery sequence before and after administration of Ibuprofen over 24 hours. The partition coefficient was calculated as $\Delta R 1_{\text {myocardium }} / \Delta R 1_{\text {blood, }}$ where $R 1=1 / T 1$.

Results: In vitro no significant correlation was found between relaxivity and Ibuprofen concentration, neither in absence $(r=-0.15, p=0.40)$ nor in presence of albumin $(r=-0.32, p=0.30)$. In vivo there was no significant difference in post contrast $\mathrm{T} 1$ times of myocardium and blood, respectively and also in the partition coefficient between exam 1 and 2 ( $p>0.05$ ). There was good agreement of the $\mathrm{T} 1$ times of myocardium and blood and the partition coefficient, respectively between exam 1 and 2 .
\end{abstract}

Conclusions: Contrast enhanced T1 mapping is unaffected by co-medication with the protein binding substance Ibuprofen and has an excellent reproducibility.

Keywords: Gadobenate dimeglumine, Ibuprofen, Interaction, CMR, Diffuse myocardial fibrosis

\section{Background}

It has been demonstrated that $\mathrm{T} 1$ time as measured by $\mathrm{T} 1$ mapping by means of cardiovascular magnetic resonance (CMR) correlates with histologically proven fibrosis.[1] Post-contrast T1 values have been shown to be altered in various cardiac diseases such as systemic lupus erythematosus [2], cardiac amyloidosis [3], chronic aortic regurgitation [4], adult congenital heart disease [5], and diabetic cardiomyopathy [6,7]. Since the concentration of the gadolinium based contrast agent is directly

\footnotetext{
* Correspondence: nadine.kawel@gmx.de

${ }^{1}$ Department of Radiology, University Hospital Basel, Petersgraben 4, Basel 4031, Switzerland

Full list of author information is available at the end of the article
}

related to the difference between pre-contrast and postcontrast reciprocal values of $\mathrm{T} 1(\Delta \mathrm{R} 1)$ and based on the assumption of a steady state of the concentration of the gadolinium based contrast agent between the extracellular space and the blood pool, the extracellular volume fraction (ECV) which is directly related to the collagen content can be quantified by calculating the ratio of preand post-contrast reciprocal values of $\mathrm{T} 1$ measured in blood and myocardium corrected for the hematocrit $[\mathrm{ECV}=\Delta \mathrm{R} 1$ myocardium $/ \Delta \mathrm{R} 1$ blood $*$ (1-hematocrit) $]$. Calculation of the partition coefficient (Lambda) is identical to the ECV except that it is not corrected for the hematocrit. Messroghli et al. demonstrated a correlation 
between ECV and the collagen volume fraction quantified by histologic analysis.[8] Wong et al. recently identified the predictive value of expansion of the extracellular matrix as calculated by ECV and all cause mortality as well as a composite end point of death, cardiac transplant and left ventricular assist device. [9] T1 mapping and calculation of the imaging biomarker ECV and the partition coefficient, respectively is a promising technique that might replace invasive myocardial biopsy in diagnosing diffuse myocardial fibrosis and might be a useful tool to monitor therapy. Advantages of CMR T1 mapping as compared to myocardial biopsy are 1) noninvasiveness and therefore the possibility to repeat measurements for treatment monitoring, 2) large measurement volume rather than small biopsy samples and 3 ) the fact that no ionizing radiation is required.

T1 mapping is technically demanding since technical, physiological, and biochemical factors can interfere with T1 measurements. For example renal function as reflected by glomerular filtration rate is of relevance [10]. Moreover, contrast material dose, relaxivity, biodistribution, clearance, interaction with plasma proteins, and interference with co-medication must be taken into consideration.

It has been demonstrated that both the $\mathrm{T} 1$ relaxation time and the partition coefficient lambda (calculated by the change in relaxation rate of blood and myocardium) vary with relaxivity and distribution properties of the contrast material [11]. Gadobenate dimeglumine (Gd-BOPTA, Multihance ${ }^{\circ}$ ) has some weak protein binding capacities and higher molar relaxivity in plasma/blood as compared to other extracellular gadolinium based contrast agents leading to a shorter T1 time [12-14]. Indirectly, rise of molar relaxivity is linked to protein interaction of the gadolinium based contrast agent influencing also its distribution with reduction of extravasation or prolongation of elimination half-time as shown with gadofosveset trisodium [15]. Other gadolinium based contrast agents also have protein binding capacity but to a lesser extent [16].

Hypothetically co-administration of another drug with a high protein binding capacity might compete with Gd-BOPTA and reduce its bound fraction, therefore altering $\mathrm{T} 1$ time. It is well known in pharmacology that albumin binding drugs potentially can interfere with each other due to this interaction with the binding sites on the albumin. This can also be explained by the fact, that the bound fraction (often $>99 \%$ ) usually is not active while the non-bound fraction (1\%) is active and can extravasate. Both effects might as well play a role when studying the relaxivity effects with Gd-BOPTA and the T1 mapping. From our perspective these potential interactions must be studied more in detail and are of high clinical interest, both from a mechanistic understanding but also clinically speaking.
Therefore the aim of this study was to evaluate the interference of a typical protein binding drug (Ibuprofen) with Gd-BOPTA with respect to T1 times in-vitro and in-vivo.

Ibuprofen was chosen since it is a common drug in widespread use available without prescription and known to have a high protein binding capacity. Moreover, the dosage in $\mathrm{mg}$ and moles compared to other non-steroidal anti-inflammatory drugs is quite high.

\section{Methods}

\section{In vitro sample preparation}

A total of 50 vials were prepared by diluting Gd-BOPTA (Gadobenate dimeglumine, Multihance, Bracco Imaging, Milan, Italy), Ibuprofen and human albumin in physiologic $\mathrm{NaCl}$ solution. The vials contained all combinations of the following dilutions: Gd-BOPTA corresponding to Gadolinium concentrations of $0,2,4,8$ and $16 \mathrm{mmol} / \mathrm{l}$, Ibuprofen in concentrations of $0,100 \mathrm{mg} / \mathrm{l}(=0.48 \mathrm{mmol} / \mathrm{l})$, $200 \mathrm{mg} / \mathrm{l} \quad(=0.97 \mathrm{mmol} / \mathrm{l}), \quad 400 \mathrm{mg} / \mathrm{l} \quad(=1.94 \mathrm{mmol} / \mathrm{l}) \quad$ and $1000 \mathrm{mg} / \mathrm{l}(=4.85 \mathrm{mmol} / \mathrm{l})$, Albumin in concentrations of 0 and $4 \mathrm{~g} / \mathrm{dl}$ (according to the physiologic concentration of human serum albumin of adults).

\section{Study subjects}

10 volunteers ( 5 men; mean age $\pm \mathrm{SD}, 31 \pm 6.3$ years) were included in the in vivo part of the study. All volunteers were healthy subjects without a known cardiovascular disease or systemic conditions and were not on regular medication. All volunteers had a normal creatinine value indicating a normal renal function and no signs of a dyslipoproteinemia as assessed by serum electrophoresis and immunfixation for Bence-Jones proteins in urine. All study participants signed informed consent in this institution review board approved study.

\section{Image acquisition - in vitro}

Samples were placed in a water container and imaged on a $1.5 \mathrm{~T}$ clinical magnet (Avanto, Siemens Medical Solutions, Erlangen, Germany) in order to establish T1 values. Samples were scanned with a coronal 2D spin echo sequence at multiple TRs with the following parameters: TE $6.2 \mathrm{~ms}$; bandwidth $781 \mathrm{~Hz} /$ pixel; slice thickness $10 \mathrm{~mm}$; field of view $300 \times 206 \mathrm{~mm}$; matrix $256 \mathrm{x}$ 176; TR 20, 25, 30, 40, 50, 75, 100, 150, 200, 250, 300, 400, 500, 1000, 1500, 2000, $4000 \mathrm{~ms}$.

\section{Image acquisition - in vivo}

Volunteers were imaged on a $1.5 \mathrm{~T}$ magnet (Espree, Siemens Medical Solutions, Erlangen, Germany). For T1 mapping a modified look-locker inversion-recovery (MOLLI) sequence was used to acquire images at midventricular short axis pre contrast and every 5 minutes between 5 and 60 minutes after intravenous bolus 
administration of $0.15 \mathrm{mmol} / \mathrm{kg}$ Gd-BOPTA. The MOLLI sequence acquired 11 images at different inversion times using the following scan parameters: TE/TR $1.06 \mathrm{~ms} / 2.5 \mathrm{~ms}$; flip angle $35^{\circ}$; bandwidth $1002 \mathrm{~Hz} /$ pixel; slice thickness $8 \mathrm{~mm}$; field of view $340 \times 255 \mathrm{~mm}$; matrix 192 x 138; TI initial 100ms; TI increment 80ms.[17]All volunteers were scanned twice at intervals of 24 hours. Immediately after the first scan $800 \mathrm{mg}$ Ibuprofen (Irfen ${ }^{\circ}-800$ retard, Mepha Pharma AG, Switzerland) was administered orally and again after 12 hours and 4 hours prior to the second scan. Ibuprofen was taken at least 30 minutes preprandial or 2 hours postprandial.

\section{Image analysis - in vitro}

T1 values were calculated for each sample by a pixelwise fitting of the signal intensities, directly on the scanner, by means of a custom reconstruction procedure [18]. The images were then transferred to a personal computer and circular ROIs were drawn on the sections of the tubes in order to obtain the average T1 values. For each Gd-BOPTA dilution series, the relaxivity value $\mathrm{r} 1$ was calculated as the slope of the linear fitting of the inverse of the T1 times, obtaining in the end 10 relaxivity values for Gd-BOPTA in presence of the different Ibuprofen concentrations, with and without albumin.

\section{Image analysis - in vivo}

In vivo T1 maps were generated using MRmap [19] and transferred to QMass V.7.2 (Medis Medical Imaging Systems, Netherlands). Left ventricular endocardial and epicardial contours were drawn manually while segments were defined automatically. T1 time was determined for each segment (American Heart Association segments 7-12 [20]) separately and also calculated for the entire slice as the mean value of all segments excluding segments with severely impaired image quality. T1 time of blood was also measured. Measurements were obtained in the LV cavity taking care to avoid inclusion of the papillary muscles using a region of interest (ROI) covering an area of $3-4 \mathrm{~cm}^{2}$. Heart rate correction as it has been suggested for studies using $3 \mathrm{~T}$ scanners was not performed since differences for shorter $\mathrm{T} 1$ times at normal heart rates are very small.[21] The partition coefficient which reflects the change in relaxation rate of myocardium and blood was approximated by $\Delta \mathrm{R} 1_{\text {myocardium }} /$ $\Delta \mathrm{R} 1_{\text {blood, }}$, where $\mathrm{R} 1=1 / \mathrm{T} 1$ [22].

\section{Statistical analysis - in vitro}

Relaxivity values in presence or absence of albumin were compared by a paired t-test to establish the effect of protein binding on the relaxivity of Gd-BOPTA. Correlation between the calculated relaxivity values and Ibuprofen concentration was evaluated by Pearson's correlation coefficient, separately in presence or absence of albumin.

\section{Statistical analysis - in vivo}

Statistical analysis was performed using PASW (SPSS) (version 19) and SAS (version 9.2) statistical software. A p-value $<0.05$ was considered statistically significant.

The continuous variables, T1 time and lambda, are expressed as mean $\pm \mathrm{SD}$ and were compared using student's paired t-test for pre contrast values of $\mathrm{T} 1$ since data was normally distributed (KolmogorovSmirnov test). A linear mixed-model analysis was performed with the log transformed T1 times for blood and myocardium to compare $\mathrm{T} 1$ time of both exams after contrast administration. Linear mixed-model analysis with the partition coefficient was also performed. Both sets of models evaluated group, time and groupby-time interaction by study subjects as random effects. Correlation between T1 times and the partition coefficient, respectively acquired in exam 1 and 2 was assessed by calculating the intraclass correlation coefficient (ICC) using a two-way random model and agreement was assessed by generating Bland Altman plots.

\section{Results}

T1-Relaxivity values of the in vitro experiment with GdBOPTA (Figure 1) were found to be significantly different in presence $\left(9.24 \pm 0.48 \mathrm{mmol}^{-1} \mathrm{~s}^{-1} \mathrm{l}\right)$ or absence $\left(7.22 \pm 0.28 \mathrm{mmol}^{-1} \mathrm{~s}^{-1} \mathrm{l}\right)$ of albumin $(\mathrm{p}=0.001)$. However, no significant correlation was found between relaxivity and Ibuprofen concentration, neither in absence $(R=-0.15$, $\mathrm{p}=0.40)$ nor in presence of albumin $(\mathrm{R}=-0.32, \mathrm{p}=0.30)$.

In the in vivo part of the study of 1560 myocardial segments evaluated ( 6 segments per slice at 13 time points in 10 subjects at 2 exams), 10 segments $(0.6 \%)$ had to be excluded from analysis related to severely impaired image quality. Mean values \pm SD of the in vivo part are shown in Table 1. Pre contrast T1 times for myocardium and blood were not significantly different between exam 1 and $2(p>0.05)$. Linear mixed model analysis did not show a group difference between exam 1 and 2 for post

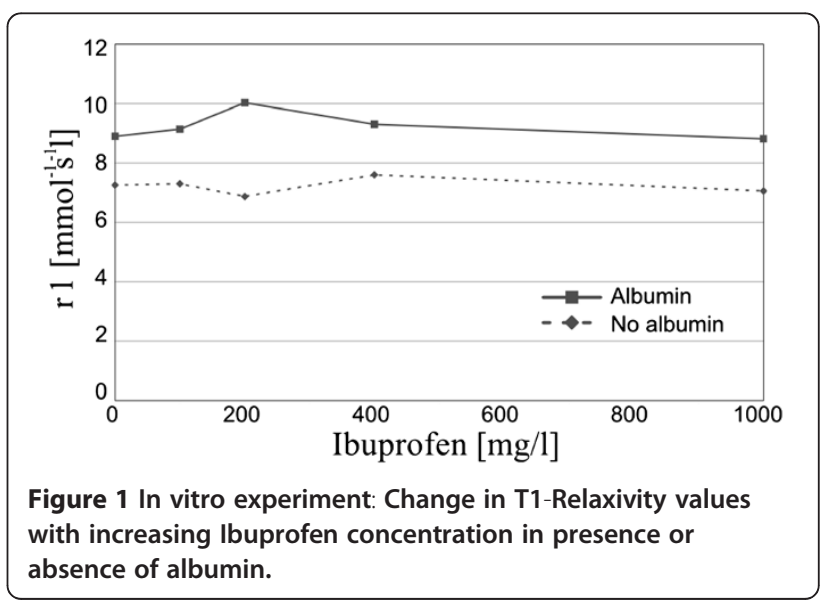


Table 1 Mean T1 times of myocardium and blood and the partition coefficient (lambda)

\begin{tabular}{|c|c|c|c|c|c|c|}
\hline \multirow[b]{2}{*}{$\min$} & \multicolumn{2}{|c|}{ T1 myocardium (ms) } & \multicolumn{2}{|c|}{ T1 blood (ms) } & \multicolumn{2}{|c|}{ Lambda } \\
\hline & Exam 1 & Exam 2 & Exam 1 & Exam 2 & Exam 1 & Exam 2 \\
\hline Pre & $1011 \pm 32$ & $1002 \pm 48$ & $1526 \pm 64$ & $1547 \pm 58$ & & \\
\hline 5 & $301 \pm 16$ & $308 \pm 22$ & $148 \pm 16$ & $155 \pm 24$ & $0.382 \pm 0.03$ & $0.386 \pm 0.04$ \\
\hline 10 & $353 \pm 21$ & $367 \pm 20$ & $199 \pm 25$ & $206 \pm 20$ & $0.420 \pm 0.04$ & $0.410 \pm 0.03$ \\
\hline 15 & $389 \pm 23$ & $367 \pm 20$ & $255 \pm 24$ & $234 \pm 21$ & $0.416 \pm 0.03$ & $0.409 \pm 0.03$ \\
\hline 20 & $411 \pm 26$ & $403 \pm 23$ & $246 \pm 29$ & $253 \pm 23$ & $0.423 \pm 0.03$ & $0.411 \pm 0.03$ \\
\hline 25 & $432 \pm 27$ & $425 \pm 18$ & $265 \pm 30$ & $272 \pm 23$ & $0.424 \pm 0.03$ & $0.411 \pm 0.03$ \\
\hline 30 & $450 \pm 22$ & $445 \pm 16$ & $283 \pm 31$ & $290 \pm 23$ & $0.426 \pm 0.03$ & $0.419 \pm 0.03$ \\
\hline 35 & $465 \pm 25$ & $460 \pm 15$ & $300 \pm 33$ & $306 \pm 24$ & $0.434 \pm 0.03$ & $0.428 \pm 0.03$ \\
\hline 40 & $475 \pm 22$ & $479 \pm 21$ & $315 \pm 33$ & $321 \pm 25$ & $0.442 \pm 0.04$ & $0.440 \pm 0.04$ \\
\hline 45 & $488 \pm 27$ & $500 \pm 17$ & $329 \pm 35$ & $338 \pm 26$ & $0.444 \pm 0.03$ & $0.432 \pm 0.03$ \\
\hline 50 & $502 \pm 27$ & $509 \pm 18$ & $344 \pm 37$ & $351 \pm 28$ & $0.446 \pm 0.04$ & $0.440 \pm 0.04$ \\
\hline 55 & $513 \pm 26$ & $525 \pm 14$ & $358 \pm 38$ & $364 \pm 29$ & $0.449 \pm 0.04$ & $0.431 \pm 0.04$ \\
\hline 60 & $524 \pm 25$ & $537 \pm 16$ & $373 \pm 38$ & $379 \pm 31$ & $0.454 \pm 0.04$ & $0.432 \pm 0.04$ \\
\hline
\end{tabular}

$\min =$ time after contrast administration.

contrast T1 values of myocardium, blood and the partition coefficient. Mean T1 times of myocardium and blood and also the mean partition coefficient increased over time $(\mathrm{p}<0.001)$ between 5 and 60 minutes after contrast administration. The mean increase of the partition coefficient between 5 and 60 minutes after contrast administration was 19\% (exam 1) and 12\% (exam 2), respectively.

The ICC for measurements of T1-time of myocardium and blood, respectively was 1.0 and for the partition coefficient it was 0.82 . Bland Altman plots demonstrate a good agreement for the measurements obtained in both exams (Figure 2).

\section{Discussion}

We studied the effect of co-medication with a typical protein binding drug (Ibuprofen) on contrast enhanced T1 mapping. Most important findings can be summarized as follows: 1.) Ibuprofen did not significantly affect T1 times of myocardium and blood when using a gadolinium based contrast agent with protein binding capacity (Gd-BOPTA) 2.) Correlations of $\mathrm{T} 1$ times of myocardium and blood and partition coefficient between exam 1 and 2 were excellent.

Injected intravenously the extracellular gadolinium based contrast agent Gd-BOPTA is known to have a transient and weak interaction with plasma proteins, mainly human serum albumin (HSA) [23]. The aromatic tail of Gd-BOPTA enables binding to hydrophobic pockets on the HSA surface [24]. Bound to HSA, the Gd-BOPTA molecule has a lower molecular tumbling rate and a longer rotational MR correlation time resulting in an increased relaxivity [25]. Relaxation rate increases exponentially with increasing HSA concentration in a physiological range [24]. The protein bound fraction of Gd-BOPTA is only about $10 \%$ [12].

We hypothesized that the protein bound fraction might be smaller and subsequently relaxivity lower resulting in longer T1 times for myocardium and blood post contrast administration when another drug with protein binding capacity is administered. Results of the in vivo and the in vitro part of the study demonstrated that T1 times were not affected by Ibuprofen. The reason might be a stronger binding to HSA of Gd-BOPTA compared to Ibuprofen. Moreover, it should be emphasized that relaxivity depends on a large variety of dynamic and structural factors which in this experiment could not be further elucidated [26]. In the present case it seems that inner-sphere water exchange which is the most important relaxivity influencing factor is definitely much more affected by ligand-protein interaction than by drug-drug or drug-protein interaction. Our in vitro experiments thus confirm, that there is no clear negative affection of relaxivity even at equimolar concentrations of Ibuprofen and Gd-BOPTA. Further studies are warranted to better understand the dynamics of waterexchange besides hydration of gadolinium complexes in the presence of various protein binding drugs in a proteinous environment. In fact, albumin not only increases the relaxivity of gadolinium complexes in the blood pool, but also emerges as a versatile carrier for a long list of therapeutic and diagnostic agents in pathologies such as diabetes, cancer, rheumatoid arthritis and infectious diseases [27]. Displacement of drugs from their plasma binding increases the unbound drug fraction potentially increasing drug effect or potential toxic effects, so that 


\section{A - Bias $11 \mathrm{~ms}=95 \%$ Limits of agreement (3ms to $18 \mathrm{~ms})$}

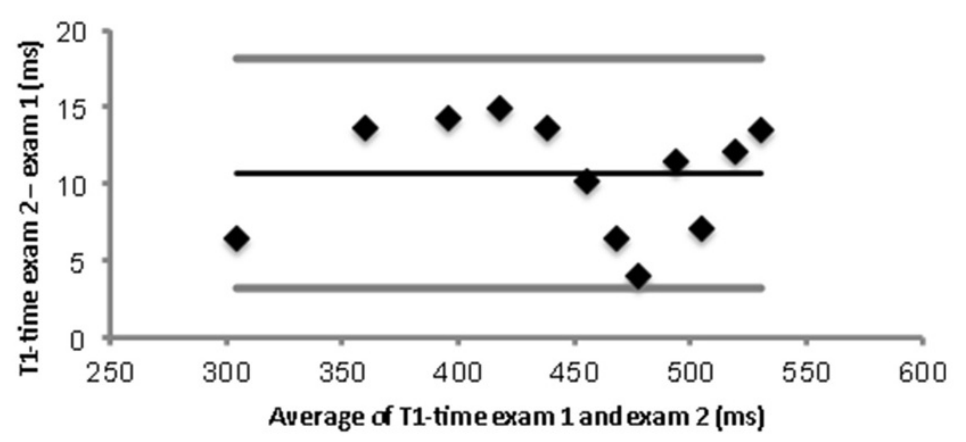

B - Bias (7.2ms) $\longrightarrow 95 \%$ Limits of agreement (4.8ms to $9.5 \mathrm{~ms})$
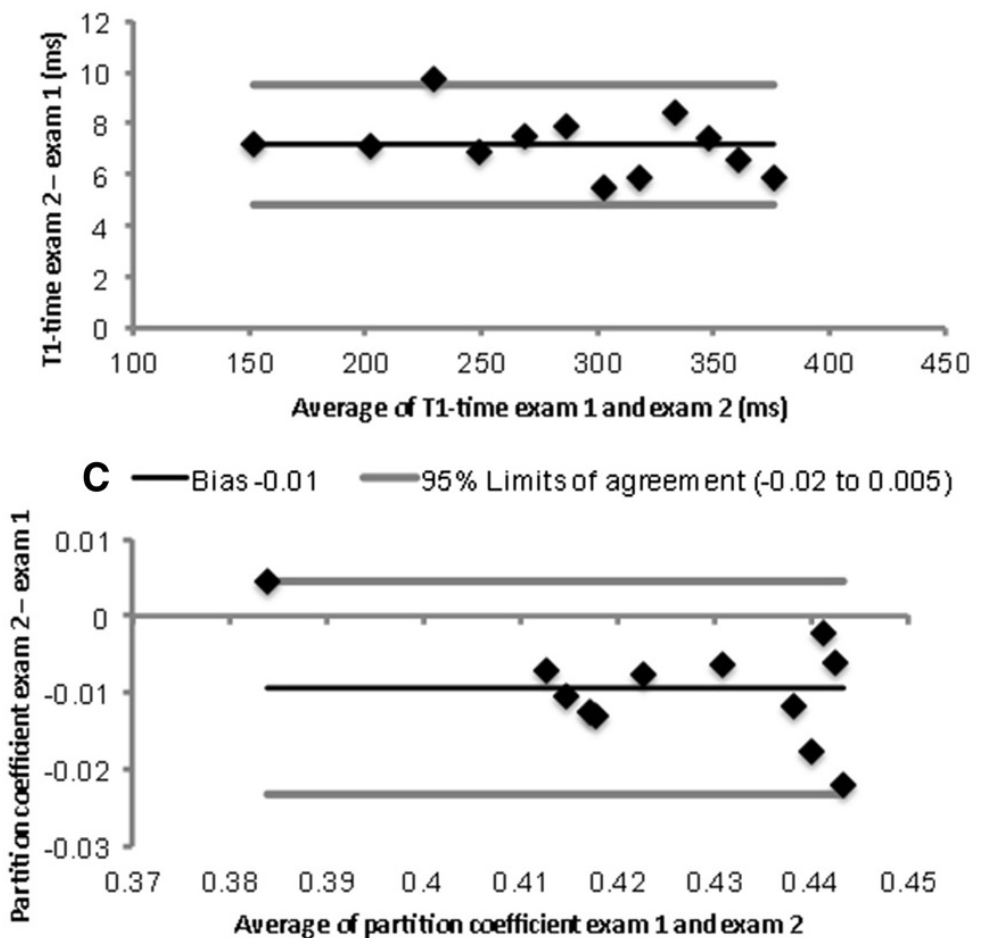

Figure 2 Agreement of T1 times of myocardium (A), blood (B) and the partition coefficient (C), respectively between exam 1 and 2.

protein-binding of drugs and drug-drug interactions are of high pharmacological interest. Gadolinium complexes with a high HSA interaction, at least in theory, could be used to visualize such effects, presuming that the drug interferes sufficiently with the Gd-complex - albumin binding sites, which was not the case in the present model.

In the current study we further demonstrated an excellent correlation and good agreement, respectively between the two exams for pre and post contrast T1 values of myocardium and blood, respectively and the partition coefficient that makes it a valuable tool for serial exams and intra-individual comparisons. This result is in agreement with a study by Messroghli et al. who found a high reproducibility of pre and post contrast myocardial T1 times [28].

According to a previous publication, we also detected a statistically significant increase of the partition coefficient over time indicating that no equilibrium establishes between the contrast in the intravascular and the interstitial space presumably related to renal excretion and distribution into other spaces such as bone and synovial fluid over the $60 \mathrm{~min}$ observation time [29]. However, the increase of the partition coefficient between 10 and 30 min after contrast administration, which would usually be the time span where T1 mapping would be acquired in a clinical protocol, was only 0.01 . Therefore the difference might not be clinically relevant. 
A limitation of the study is the fact that we tested only for the effect of Ibuprofen on T1 time. Other substances with high protein binding capacity such as Diltiazem, Propranolol, Itraconazole or Sufentanil were not evaluated [27]. However, we intended to test a substance that is easily available without prescription, not harmful to volunteers and commonly used. Another limitation might be the fact that we studied the effect of a protein binding substance on the partition coefficient but not on the ECV. However, since calculation of the partition coefficient and ECV is identical, expect for the fact that the latter is multiplied by a constant (1-hematocrit), this should be irrelevant regarding the evaluation of the effect of Ibuprofen.

\section{Conclusions}

In conclusion, contrast enhanced $\mathrm{T} 1$ mapping of Gd-BOPTA is unaffected by co-medication with the protein binding substance Ibuprofen in vitro and in vivo and has an excellent reproducibility.

\section{Competing interests}

One of the co-authors, JMF, is a consultant for Guerbet Pharmaceutical Company Switzerland.

\section{Authors' contribution}

NK: study design, data acquisition, data analysis, data interpretation, manuscript drafting; FS: study design, data acquisition, data analysis, data interpretation, manuscript drafting; TH: data acquisition, manuscript revision; JMF: study design, phantom preparation, data interpretation, manuscript revision; JB: principal investigator, study design, data interpretation, manuscript revision. All authors read and approved the final manuscript.

\section{Acknowledgement}

This study was sponsored in part by Guerbet Pharmaceutical Company Switzerland.

\section{Author details}

${ }^{1}$ Department of Radiology, University Hospital Basel, Petersgraben 4, Basel 4031, Switzerland. ${ }^{2}$ Radiological Physics, University Hospital Basel, Petersgraben 4, Basel 4031, Switzerland. ${ }^{3}$ Scientific Affairs, Guerbet Switzerland, Winterthurerstrasse 92, Zurich 8006, Switzerland.

Received: 25 April 2012 Accepted: 11 October 2012

Published: 15 October 2012

\section{References}

1. Iles L, Pfluger H, Phrommintikul A, Cherayath J, Aksit P, Gupta SN, Kaye DM, Taylor AJ: Evaluation of diffuse myocardial fibrosis in heart failure with cardiac magnetic resonance contrast-enhanced T1 mapping. J Am Coll Cardiol 2008, 52:1574-1580

2. Been M, Thomson BJ, Smith MA, Ridgway JP, Douglas RH, Best JJ, Muir AL: Myocardial involvement in systemic lupus erythematosus detected by magnetic resonance imaging. Eur Heart J 1988, 9:1250-1256.

3. Maceira AM, Joshi J, Prasad SK, Moon JC, Perugini E, Harding I, Sheppard MN, Poole-Wilson PA, Hawkins PN, Pennell DJ: Cardiovascular magnetic resonance in cardiac amyloidosis. Circulation 2005, 111:186-193.

4. Sparrow P, Messroghli DR, Reid S, Ridgway JP, Bainbridge G, Sivananthan MU: Myocardial T1 mapping for detection of left ventricular myocardial fibrosis in chronic aortic regurgitation: pilot study. AJR Am J Roentgenol 2006, 187:W630-W635.

5. Broberg CS, Chugh SS, Conklin C, Sahn DJ, Jerosch-Herold M: Quantification of diffuse myocardial fibrosis and its association with myocardial dysfunction in congenital heart disease. Circ Cardiovasc Imaging 2010, 3:727-734.
6. Jellis C, Martin J, Narula J, Marwick TH: Assessment of nonischemic myocardial fibrosis. J Am Coll Cardiol 2010, 56:89-97.

7. Ng AC, Auger D, Delgado V, van Elderen SG, Bertini M, Siebelink HM, van der Geest RJ, Bonetti C, van der Velde ET, de Roos A, et al:: Association between diffuse myocardial fibrosis by cardiac magnetic resonance contrast-enhanced t1 mapping and subclinical myocardial dysfunction in diabetic patients: a pilot study. Circ Cardiovasc Imaging 2012, 5:51-59.

8. Messroghli D, Nordmeyer S, Dietrich T, Dirsch O, Kaschina E, Savvatis K: D OHI, Klein C, Berger F, Kuehne T: Assessment of Diffuse Myocardial Fibrosis in Rats Using Small Animal Look-Locker Inversion Recovery (SALLI) T1 Mapping. Imaging: Circ Cardiovasc; 2011.

9. Wong TC, Piehler K, Meier CG, Testa SM, Klock AM, Aneizi AA, Shakesprere J, Kellman P, Shroff SG, Schwartzman DS, et al.: Association between extracellular matrix expansion quantified by cardiovascular magnetic resonance and short-term mortality. Circulation 2012, 126:1206-1216.

10. Gai N, Turkbey EB, Nazarian S, van der Geest RJ, Liu CY, Lima JA, Bluemke DA: T(1) mapping of the gadolinium-enhanced myocardium: Adjustment for factors affecting interpatient comparison. Magn Reson Med 2011, 65:1407-1415

11. Kawel N, Nacif M, Santini F, Liu S, Bremerich J, Arai AE, Bluemke DA: Partition coefficients for gadolinium chelates in the normal myocardium: Comparison of gadopentetate dimeglumine and gadobenate dimeglumine. J Magn Reson Imaging 2012, 36:733-777.

12. Aime $\mathrm{S}$, Caravan P: Biodistribution of gadolinium-based contrast agents, including gadolinium deposition. J Magn Reson Imaging 2009, 30:1259-1267

13. Rohrer M, Bauer H, Mintorovitch J, Requardt M, Weinmann HJ: Comparison of magnetic properties of MRI contrast media solutions at different magnetic field strengths. Invest Radiol 2005, 40:715-724.

14. Spinazzi A, Lorusso V, Pirovano G, Kirchin M: Safety, tolerance, biodistribution, and MR imaging enhancement of the liver with gadobenate dimeglumine: results of clinical pharmacologic and pilot imaging studies in nonpatient and patient volunteers. Acad Radiol 1999, 6:282-291.

15. Lauffer RB, Parmelee DJ, Dunham SU, Ouellet HS, Dolan RP, Witte S, McMurry TJ, Walovitch RC: MS-325: albumin-targeted contrast agent for MR angiography. Radiology 1998, 207:529-538.

16. Wang $Y$, Spiller M, Caravan P: Evidence for weak protein binding of commercial extracellular gadolinium contrast agents. Magn Reson Med 2010, 63:609-616.

17. Messroghli DR, Greiser A, Frohlich M, Dietz R, Schulz-Menger J: Optimization and validation of a fully-integrated pulse sequence for modified look-locker inversion-recovery (MOLLI) T1 mapping of the heart. J Magn Reson Imaging 2007, 26:1081-1086.

18. Santini FPS, Scheffler K: IceLuva: A scripting framework for MR image reconstruction based on free software. Concepts in Magnetic Resonance Part B: Magnetic Resonance Engineering 2011, 39B:1-10.

19. Messroghli DR, Rudolph A, Abdel-Aty H, Wassmuth R, Kuhne T, Dietz R, Schulz-Menger J: An open-source software tool for the generation of relaxation time maps in magnetic resonance imaging. BMC Med Imaging 2010, 10:16.

20. Cerqueira MD, Weissman NJ, Dilsizian V, Jacobs AK, Kaul S, Laskey WK, Pennell DJ, Rumberger JA, Ryan T, Verani MS: Standardized myocardial segmentation and nomenclature for tomographic imaging of the heart: a statement for healthcare professionals from the Cardiac Imaging Committee of the Council on Clinical Cardiology of the American Heart Association. Circulation 2002, 105:539-542.

21. Lee J, Liu S, Nacif MS, Ugander M, Kawel N, Sibley CT, Kellman P, Arai A, Bluemke DA: Myocardial T1 and Extracellular Volume Fraction Mapping at 3 Tesla. Journal of cardiovascular magnetic resonance: official journal of the Society for Cardiovascular Magnetic Resonance 2011, 13:75.

22. Sharma P, Socolow J, Patel S, Pettigrew Rl, Oshinski JN: Effect of Gd-DTPA-BMA on blood and myocardial T1 at $1.5 \mathrm{~T}$ and $3 \mathrm{~T}$ in humans. J Magn Reson Imaging 2006, 23:323-330.

23. Spinazzi A, Lorusso V, Pirovano G, Taroni P, Kirchin M, Davies A: Multihance clinical pharmacology: biodistribution and MR enhancement of the liver. Acad Radiol 1998, 5 Suppl 1:S86-S89. discussion S93-S84.

24. Giesel FL, von Tengg-Kobligk H, Wilkinson ID, Siegler P, von der Lieth CW, Frank M, Lodemann KP, Essig M: Influence of human serum albumin on longitudinal and transverse relaxation rates ( $r 1$ and $r 2)$ of magnetic resonance contrast agents. Invest Radiol 2006, 41:222-228. 
25. Vander Elst L, Laurent S, Bintoma HM, Muller RN: Albumin-bound MRI contrast agents: the dilemma of the rotational correlation time. MAGMA 2001, 12:135-140.

26. Caravan P: Protein-targeted gadolinium-based magnetic resonance imaging (MRI) contrast agents: design and mechanism of action. Acc Chem Res 2009, 42:851-862.

27. Elsadek B, Kratz F: Impact of albumin on drug delivery - New applications on the horizon. J Control Release 2012, 157:4-28

28. Messroghli DR, Plein S, Higgins DM, Walters K, Jones TR, Ridgway JP, Sivananthan MU: Human myocardium: single-breath-hold MR T1 mapping with high spatial resolution-reproducibility study. Radiology 2006, 238:1004-1012.

29. Kawel N, Nacif M, Zavodni A, Jones J, Liu S, Sibley CT, Bluemke DA: T1 mapping of the myocardium: intra-individual assessment of post-contrast T1 time evolution and extracellular volume fraction at $3 \mathrm{~T}$ for Gd-DTPA and Gd-BOPTA. J Cardiovasc Magn Reson 2012, 14:26.

doi:10.1186/1532-429X-14-71

Cite this article as: Kawel et al.: The protein binding substance Ibuprofen does not affect the T1 time or partition coefficient in contrast-enhanced cardiovascular magnetic resonance. Journal of Cardiovascular Magnetic Resonance 2012 14:71.

\section{Submit your next manuscript to BioMed Central and take full advantage of:}

- Convenient online submission

- Thorough peer review

- No space constraints or color figure charges

- Immediate publication on acceptance

- Inclusion in PubMed, CAS, Scopus and Google Scholar

- Research which is freely available for redistribution 